\section{L'Actualité économique}

L'ACTUALITÉ ÉCONOMIQUE

\section{Les lignes directrices de mon oeuvre}

\section{Maurice Allais}

Volume 65, numéro 3, septembre 1989

URI : https://id.erudit.org/iderudit/601494ar

DOI : https://doi.org/10.7202/601494ar

Aller au sommaire du numéro

Éditeur(s)

HEC Montréal

ISSN

0001-771X (imprimé)

1710-3991 (numérique)

Découvrir la revue

Citer cet article

Allais, M. (1989). Les lignes directrices de mon oeuvre. L'Actualité économique, 65(3), 323-345. https://doi.org/10.7202/601494ar
Ce document est protégé par la loi sur le droit d'auteur. L'utilisation des services d'Érudit (y compris la reproduction) est assujettie à sa politique d'utilisation que vous pouvez consulter en ligne.

https://apropos.erudit.org/fr/usagers/politique-dutilisation/ 


\title{
LES LIGNES DIRECTRICES DE MON GEUVR**
}

\author{
Maurice ALLAIS \\ École Nationale Supérieure des Mines de Paris \\ et Centre National de la Recherche Scientifique - France
}

Le Prix Nobel qui m'a été attribué par l'Académie Royale des Sciences de Suède représente pour moi un très grand honneur que je ressens profondément.

Je me sens d'autant plus honoré que c'est la première fois, cette année, que le Lauréat pour les sciences économiques prononce sa Conférence Nobel devant l'Académie des Sciences.

Il est devenu de tradition que le Lauréat présente ses principales contributions en liaison avec la motivation du Prix, en l'espèce « mes travaux de pionnier sur la théorie des marchés et l'utilisation efficace des ressources». Je me permettrai d'interpréter cette motivation au sens large, c'est-à-dire comme relative à toutes les conditions susceptibles d'assurer une efficacité maximale de l'économie pour satisfaire au mieux les besoins des hommes au regard des ressources limitées dont ils disposent.

I. MOTIVATIONS DE MA CARRIÈRE D'ÉCONOMISTE, MON OUVRAGE DE 1943, ET SES PROLONGEMENTS ULTÉRIEURS

\section{Motivations de ma carrière d'économiste}

Mes contributions à la science économique forment un tout, et elles ne peuvent être comprises qu'au regard des motivations dont a résulté ma carrière d'économiste.

Passionné par l'Histoire au cours de mes études secondaires, puis par la Physique et la Mécanique à l'École Polytechnique, je suis finalement entré dans le service actif des Mines en 1936.

Mais mes inclinations réelles étaient ailleurs, et isolé que j'étais dans un service provincial, je consacrais mes loisirs à différentes lectures sur la physique et les probabilités. J'entrepris même en 1939 la rédaction d'un ouvrage d'ensemble sur les probabilités.

\footnotetext{
*Conférence Nobel prononcée devant l'Académie Royale des Sciences de Suède, Stockholm, le 9 décembre 1988, et au congrès annuel de la Société canadienne de science économique, Mont-Rolland, Québec, le 24 mai 1989. @ FONDATION NOBEL 1988.
} 
Puis sont venues la guerre et la défaite de la France. En juillet 1940, après ma démobilisation, je repris mes fonctions d'ingénieur dans le Service des mines, à Nantes, dans la zone occupée par l'armée allemande. Mais mes préoccupations d'avant-guerre s'étaient profondément modifiées. Pour moi, et avec toutes les illusions de la jeunesse (j'avais alors vingt-neuf ans), il était clair que ce que je pouvais faire de mieux était de contribuer à préparer l'après-guerre.

J'avais, au cours de l'été 1933, visité les États-Unis, alors au creux de la Grande Dépression, phénomène profondément étonnant auquel aucune explication communément acceptable n'avait pu être donnée. J'avais également vécu de très près les troubles sociaux qui s'étaient constatés en France à la suite des élections de 1936.

Comment mieux préparer l'après-guerre que d'essayer de résoudre le problème fondamental de toute économie : promouvoir une efficacité économique aussi grande que possible tout en assurant une répartition des revenus qui soit communément acceptable?

Ainsi, ma vocation d'économiste n'a pas été déterminée par l'école, mais par les circonstances. Elle a eu pour objet de chercher à établir les fondations sur lesquelles une politique économique et sociale pourrait être valablement édifiée.

Mon ouvrage de 1943 - À la recherche d'une discipline économique - L'économie pure

J'ai alors acheté, un peu au hasard, tous les ouvrages d'économie d'auteurs français, ou d'auteurs étrangers traduits en français, qui se trouvaient disponibles.

Là a commencé une période de ma vie qui aujourd 'hui encore me paraît presque incompréhensible. Comment m'a-t-il été possible d'écrire un ouvrage, très dense et très structuré, d'environ mille pages, « À la recherche d' une discipline économique. L'économie pure », en trente mois seulement, de janvier 1941 à juillet 1943, ouvrage auquel votre Académie s'est expressément référée dans l'exposé des motifs de sa décision de m'attribuer le Prix Nobel d'Économie 1988.

Certes, le service administratif que je dirigeais et qui couvrait cinq départements français ne fonctionnait qu'au ralenti sous l'occupation allemande, mais il me prenait au moins vingt-cinq heures par semaine. Je devais en outre faire des visites de mines et de carrières, et de fréquents déplacements à Paris, distant de quelque quatre cents kilomètres.

Je n'étais à l'époque qu'un autodidacte. En fait, toutes mes lectures économiques n'ont commencé qu'en juillet 1940. Des indications manuscrites, et notamment des dates que je portais alors sur les ouvrages que je lisais et annotais, il ressort par exemple que c'est seulement de juillet 1940 à mai 1941 que j'ai lu les ouvrages fondamentaux de Léon Walras, de Vilfredo Pareto et d'Irving Fisher, les trois grands économistes qui m'ont de loin le plus influencé.

Comment m'a-t'il été possible de rédiger un tel ouvrage dans un délai aussi court, et cela dans les conditions si difficiles des années noires de la guerre et de 
l'occupation allemande en France ? Comment ai-je pu achever l'Introduction à cet ouvrage dès le ler juillet 1941, un an seulement après ma démobilisation ? Comment ai-je pu préparer efficacement dans cette même période les matériaux des cinq autres volumes qui devaient suivre?

Comment ai-je pu parallèlement trouver un imprimeur, du papier (alors introuvable) pour l'impression, et organiser une souscription au regard du refus de tous les éditeurs de publier cet ouvrage?

Tout cela apparaît aujourd' hui totalement invraisemblable, et pourtant tout ce travail a effectivement été réalisé et mené à bien en trente mois seulement.

Rien peut-être ne peut sans doute mieux décrire l'espèce d'exaltation où je me trouvais alors que ces quelques passages d'une lettre de Leibniz : « Je voulais nager par moi-même, sans maître... Souvent à la lumière de quelques phrases au cours de mes lectures je puisais le sujet d' innombrables méditations... » Il me faut encore ajouter que, sans le connaître, je suivais de moi-même le principe fondamental d'Abel : «Ne jamais lire que les grands maîtres dans leurs travaux originaux».

Mon ouvrage de 1943 n'a été ainsi que le travail d'un amateur, mais d'un amateur passionné, et si quelqu'un pouvait à l'occasion sursauter en apprenant que 1'Académie Royale des Sciences de Suède s'est expressément référée à l'ouvrage d'un auteur qui s'est qualifié lui-même d'amateur, c'est qu'il ignorerait tout ce que les amateurs ont pu apporter à la Science dans le passé. Dans leurs rangs se trouvent en effet de très grandes figures : les Fermat, les Leibniz, les Lavoisier, les Mendel, les Pasteur, les Louis de Broglie... et une foule d'autres encore, qui, à leurs débuts, ou même pour certains d'entre eux toute leur vie, n'ont été que des amateurs. Walras et Pareto n'étaient eux-mêmes que des autodidactes et des amateurs.

S'ils sont généralement détestés des professionnels et des « establishments » de toutes sortes, les amateurs bénéficient par contre d'un avantage tout à fait exceptionnel, c'est de ne pas avoir été conditionnés par l'enseignement et la répétition incessante des «vérités établies », et d'être effectivement capables d'examiner toute question avec des yeux neufs, sans parti pris, ni préjugé.

\section{Les prolongements de mon ouvrage de 1943}

Mon ouvrage de 1943 a été à l'origine de tous mes travaux ultérieurs qui n'en ont constitué que les prolongements et les compléments dans les différents domaines de la science économique sur lesquels j'ai travaillé, et cela en poursuivant toujours un même objectif : l'établissement rigoureux d'une théorie générale en profond accord avec les données de l'observation.

Initialement, et comme il est indiqué dans ses premières pages, mon ouvrage de 1943 devait être suivi de cinq autres volumes, les trois premiers consacrés respectivement à la théorie de l'intérêt, à la théorie monétaire, et à l'économie internationale ; le quatrième consacré à l'analyse des déséquilibres de l'économie réelle ; et le cinquième enfin, d'ordre normatif, L'économie de l'avenir. 
Ce projet, très ambitieux, s'est révélé peu réaliste, et il m'a paru préférable d'y renoncer. Pour une large part, la substance des deuxième, troisième, et cinquième volumes a été publiée en 1947 dans mon ouvrage «Économie et Intérêt », auquel votre Académie s'est également expressément référée dans la motivation de sa décision. Là encore seule une souscription a permis l'édition de cet ouvrage.

En fait, toute mon œuvre n'a constitué que la réalisation progressive du programme que je m'étais fixé en 1943. Cette réalisation n'est pas encore achevée, et elle se poursuit encore aujourd'hui.

Si ma motivation initiale a été normative, si elle a correspondu pour une large part aux préoccupations exprimées par Walras dans ses «Études d'Économie Sociale », j'ai néanmoins constamment séparé avec le plus grand soin, sur le plan de l'analyse, mes travaux d'économie fondamentale de mes travaux d'économie appliquée et de politique économique.

Ma préoccupation dominante a été celle de la synthèse. Faire rentrer dans une même construction l'analyse des phénomènes réels et celle des phénomènes monétaires, associer l'analyse des conditions d'efficacité et celle de la répartition des revenus, relier étroitement l'analyse théorique et l'économie appliquée, rattacher l'économie aux autres sciences humaines, la psychologie, la sociologie et l'histoire, tels ont été constamment mes objectifs.

C'est cette préoccupation d'une conception synthétique de tous les phénomènes économiques et sociaux qui constitue le soubassement de toute mon œuvre et le lien étroit entre mes travaux d'économie théorique et ceux d'économie appliquée. C'est elle qui explique la profonde uninité sous-jacente à tous mes travaux.

Dans leur élaboration la démarche de ma pensée n'a jamais été de partir de la théorie pour aboutir aux faits, mais tout au contraire, d'essayer de dégager des faits la trame explicative sans laquelle ils apparaissent incompréhensibles et échappent à toute action efficace.

Mes travaux ont répondu au besoin que j'ai ressenti de comprendre la réalité concrète, et de donner des réponses satisfaisantes aux questions que me suggéraient les obscurités, les contradictions, et les lacunes de la littérature existante. Mon œuvre a ainsi représenté pour moi un long effort, souvent pénible, pour me dégager des chemins battus et des conceptions dominantes de mon temps.

Au début de ma carrière mon désir de comprendre a été motivé par le désir profond d'agir, par le souci d'influencer l'opinion et la politique ; cependant, progressivement, au cours des années, cette motivation est passée tout à fait au second plan, très loin derrière le désir de comprendre.

\section{CONTRIBUTIONS PRINCIPALES À LA SCIENCE ÉCONOMIQUE}

Mes contributions à l'analyse économique fondamentale ont porté essentiellement sur cinq domaines, tous liés à la recherche des conditions d'une efficacité aussi 
grande que possible de l'économie et à l'analyse de la répartition des revenus qu'elle implique, domaines sur lesquels je n'ai cessé de travailler depuis 1941 ; la théorie de l'évolution et de l'équilibre économique général, de l'efficacité maximale, et des fondements du calcul économique ; - la théorie des processus intertemporels et d'une efficacité capitalistique maximale ; - la théorie des choix aléatoires et des critères à considérer pour des décisions économiques rationnelles ; - la théorie de la monnaie, du crédit, et de la dynamique monétaire ; - la théorie des probabilités, et l'analyse des séries temporelles et de leurs composantes exogènes.

Dans tous ces domaines je pense m'être libéré des conceptions habituelles, avoir ouvert des voies originales, et dégagé de nouvelles perspectives.

Je me bornerai ici à commenter très brièvement celles de mes contributions susceptibles d'être considérées, au sens du testament d'Alfred Nobel, soit comme des apports majeurs à la science économique fondamentale, soit même, si je puis dire, comme de véritables découvertes.

Théorie de l'évolution et de l'équilibre économique général, de l'efficacité maximale, et des fondements du calcul économique

Mes travaux sur l'évolution et l'équilibre économique général, l'efficacité maximale, et les fondements du calcul économique, se sont développés en deux phases successives, de 1941 à 1966 et de 1967 à aujourd'hui.

- Pour une grande part mon ouvrage de 1943 « À la Recherche d' une Discipline Économique - L'Économie Pure » s'est centré sur la démonstration de deux propositions fondamentales : toute situation d'équilibre d'une économie de marché est une situation d'efficacité maximale, et réciproquement toute situation d'efficacité maximale est une situation d'équilibre d'une économie de marché (théorèmes d'équivalence).

Dans le cadre du modèle de l'économie de marché de Walras la démonstration rigoureuse de ces propositions, remarquablement simples et qui avaient été pressenties par les économistes classiques, soulève bien des difficultés. Je pense l'avoir donnée pour la première fois, avec une grande généralité, pour une économie considérée à un instant donné du temps, mais tenant compte de l'avenir.

Cette démonstration tient compte des conditions du second ordre et elle est affranchie de toute hypothèse irréaliste de convexité générale en ce qui concerne les champs de production. Elle met en évidence l'arbitraire de la répartition des revenus. La formulation des valeurs actualisées apparaît comme une condition d'efficacité maximale.

Dans cet ouvrage j'ai défini quatre nouveaux concepts tout à fait fondamentaux : - le concept de surface des possibilités maximales dans l' hyper-espace des indices de préférences des unités de consommation : - le concept de surplus distribuable correspondant à une modification virtuelle de l'économie à partir d'une situation donnée ; - le concept de perte, définie comme la valeur maximale 
du surplus distribuable pour toutes les modifications réalisables de l'économie qui laissent les indices de préférence des opérateurs inchangés; et le concept associé de surfaces d'égale perte dans l'hyper-espace des indices de préférence.

- Au cours de l'hiver 1966-1967 j'ai été amené à abandonner complètement le modèle général de l'économie de marché de Walras caractériséà tout moment, qu'il $y$ ait équilibre ou non, par un système unique de prix, le même pour tous les opérateurs, hypothèse tout à fait irréaliste, et à fonder la théorie de l'évolution et de l'équilibre économique général, de l'efficacité maximale, et des fondements du calcul économique, sur des bases entièrement nouvelles à partir du concept de surplus distribuable que j'avais élaboré et utilisé dans mon ouvrage de 1943 et d'un nouveau modèle, le modèle de l'économie de marchés (avec un s).

Suivant cette nouvelle approche, qui en fait était déjà largement esquissée dans de nombreux développements de mon ouvrage de 1943, toute la dynamique économique en termes réels se fonde sur la recherche, la réalisation et la répartition de surplus. Elle permet de donner des deux théorèmes d'équivalence des démonstrations remarquablement simples et totalement affranchies de toute hypothèse restrictive que ce soit, de continuité, de différentiabilité ou de convexité générale. Elle permet en outre de spécifier un système de règles du jeu dont l'application est susceptible de mener à des situations d'efficacité maximale. Il y a équilibre économique général et efficacité maximale lorsqu'il n'existe plus aucun surplus réalisable.

Cette theorie repose sur les concepts fondamentaux de surplus distribuable, de perte, et de surfaces d'efficacité maximale et d'égale perte présentés et élaborés dans mon ouvrage de 1943 ; et elle permet d'intégrer la monnaie dans la théorie de l'équilibre économique général et de l'efficacité maximale.

Dans ses lignes directrices ma théorie des surplus généralise les analyses marginalistes du XIXème siècle en considérant non plus simplement des variations différentielles, mais également des variations discrètes, et en tenant compte des interactions complexes des variations de toutes les quantités de l'ensemble de l'économie. Elle constitue en fait une synthèse de l'approche marginaliste de la causalité et de l'approche walrasienne de l'interdépendancefonctionnelle, approches complémentaires l'une de l'autre, dont j'avais déjà présenté une analyse dans un chapitre de mon ouvrage de 1943.

Non seulement cette théorie donne une représentation réaliste de la dynamique économique, dégagée de toute hypothèse inutile, mais elle permet de mieux comprendre la signification réelle du fonctionnement de l'économie sous son double aspect de gestion et de répartition qu'elle présente sous un jour entièrement nouveau.

Cette théorie se prête tout aussi bien à l'analyse des échanges internationaux qu'à celle des économies nationales, et tout aussi bien à l'analyse des économies de l'Est et du Tiers Monde qu'à l'analyse des économies occidentales. 
Je pense que ma théorie générale des surplus constitue un progrès considérable, à vrai dire tout à fait révolutionnaire, non seulement sur toutes les théories antérieures, mais également sur toutes les théories actuelles.

Théorie des processus intertemporels et d' une structure capitalistique optimale

Mon ouvrage Économie et Intérêt de 1947 a présenté une théorie générale de l'efficacité des processus intertemporels avec deux contributions originales particulièrement significatives, l'extension de la théorie de l'efficacité maximale au cas où l'on considère les différentes générations, et la théorie de la productivité capitalistique.

- Tout d'abord lorsque l'on tient compte des générations futures, des circonstances essentielles apparaissent qui aujourd' hui encore sont très généralement ignorées dans la littérature. Essentiellement cette analyse montre que si l'unicité des taux d'intérêt est une condition nécessaire d'efficacité dans le secteur de la production, il n'en est pas de même pour l'ensemble de l'économie.

Il résulte de là que la théorie classique, suivant laquelle l'équilibre de l'offre et de la demande de capital conduit à un optimum intrinsèque, ne saurait être admise, et que notamment une politique d'épargne obligatoire en vue de la vieillesse est tout à fait compatible avec les conditions d'une efficacité maximale intertemporelle.

- En second lieu ma théorie de la productivité capitalistique maximale analyse l'influence du caractère plus ou moins indirect des processus de production sur le niveau du revenu national réel, lui-même fonction du taux d'intérêt salarial. Cette théorie a donné, pour la première fois à ma connaissance dans la littérature, la démonstration rigoureuse de l'existence d' une situation de " maximum maximorum " pour un régime permanent. Cette situation correspond à un taux d'intérêt salarial nul.

Cette théorie repose sur deux nouveaux concepts : celui de revenu originaire (valeur globale des salaires et des rentes foncières) et celui de fonction caractéristique, représentative des processus intertemporels de production, concepts que j'ai élaborés en généralisant les analyses pénétrantes de Georges Bousquet, ellesmêmes inspirées de celles de Stanley Jevons.

Ce sont là les bases sur lesquelles se sont fondés tous mes travaux ultérieurs. Dans le cas d'un processus dynamique j'ai montré en 1961 que la situation de " maximum maximorum » correspond à l'égalité du taux d'intérêt salarial et du taux de croissance du revenu originaire, égalité qu' il est convenu d'appeler aujourd' hui la "golden rule of accumulation". Je pense en avoir donné la première démonstration générale et rigoureuse.

À ma connaissance, de toutes les théories des processus capitalistiques dynamiques, celle que j'ai présentée est la seule qui se prête à des applications numériques. Elle est entièrement confirmée par les données de l’observation. 
Théorie des choix aléatoires et des critères à considérer pour des décisions rationnelles

Ma théorie des choix aléatoires est partie d'une double motivation : - le souci d'étendre les théories de l'équilibre économique général et de l'efficacité maximale au cas d'une économie aléatoire ; - une réflexion critique sur la Theory of Games de 1947 de Von Neumann-Morgenstern, et sur les critères à considérer pour des décisions économiques rationnelles.

- Dans un mémoire de 1952 j'ai montré comment il est possible de tenir compte de l'incertitude de l'avenir, des champs de choix aléatoires, et des opérations relatives à la composition des risques, (transposition dans le cas de l'aléatoire des fonctions de production concernant les opérations de transformation des biens physiques), pour généraliser les théories de l'équilibre économique général et de l'efficacité maximale au cas du risque.

- Dans la Theory of Games, Von Neumann et Morgenstern ont présenté à la fois une méthode pour déterminer l'utilité cardinale et une règle rationnelle de comportement. L'une et l'autre reposent sur la considération d'un indice qu'on peut appeler indice néo-Bernoullien d'utilité. La théorie de Von Neumann-Morgenstern en démontre l'existence à partir d'un système de postulats, et ils l'identifient avec l'utilité cardinale au sens de Jevons. Selon eux, pour être rationnel, tout opérateur doit maximer l'espérance mathématique de cet indice.

Cette position m'est apparue comme inacceptable, car elle revient à négliger la distribution de probabilité des valeurs psychologiques autour de leur moyenne, ce qui précisément représente l'élément psychologique fondamental de la théorie du risque.

J'ai illustré mon argumentation par des contre-exemples dont l'un est devenu célèbre sous la dénomination de l' « Allais' Paradox ». En faitl' « Allais' Paradox » n'est paradoxal qu'en apparence, et il correspond simplement à une réalité psychologique très profonde, la préférence pour la sécurité au voisinage de la certitude.

- Afin de soumettre au contrôle de l'expérience les oppositions doctrinales quant au comportement rationnel en face de l'incertitude, j'ai procédé en 1952 à un sondage auprès d'une centaine de personnes que leur formation et leur connaissance de la théorie des probabilités permettaient de considérer comme ayant un comportement rationnel.

Ce n'est cependant qu'au cours des années 1974-1976 que j'ai pu procéder à un analyse approfondie des réponses reçues lors du sondage de 1952. Cette analyse a pleinement confirmé les conclusions de mon mémoire de 1952 en montrant que pour aucun sujet il n' existe d' indice dont la maximation de l'espérance mathématique puisse expliquer le comportement observé.

- En outre cette analyse a permis de montrer que pour tous les sujets considérés il existe effectivement un indice de valeur psychologique, ou utilité cardinale, qui peut être déterminé indépendamment de la considération de tout choix aléatoire. 
La fonction d'utilité cardinale ainsi déterminée est invariante d'un individu à l'autre dès lors que l'on considère les variations relatives de son capital, et sa connaissance permet d'analyser quantitativement des questions qui échappaient jusqu' ici à tout calcul précis, telles que la question des incidences psychologiques des transferts de richesses des plus riches aux plus pauvres, ou la question de l'estimation des incidences psychologiques des charges fiscales.

\section{Théorie de la monnaie, du crédit, et de la dynamique monétaire}

L'expérience montre qu'il ne peut y avoir ni efficacité de l'économie, ni équité de la répartition des revenus, dans une économie monétairement instable, susceptible de comporter des fluctuations de grande ampleur, telles que celles correspondant à la Grande Dépression de 1929-1934. C'est cette constatation qui m'a amené dès 1941 à réfléchir sur les phénomènes monétaires, la monnaie, le crédit, et les fluctuations conjoncturelles.

- Dans deux mémoires de 1954 et 1955, j'ai présenté un modèle explicatif non linéaire des fluctuations de la dépense globale. Ce modèle se fonde sur une équation que j'ai appelée équation fondamentale de la dynamique monétaire, et sur une formulation héréditaire de la demande et de l'offre de monnaie. Ce modèle permet d'exprimer les variations de la dépense globale à partir de la différence entre l'offre et la demande de monnaie, ces dernières étant des fonctionnelles des variations passées de la dépense globale.

On constate que pour des valeurs des paramètres voisines de celles données par l'expérience, le modèle comporte des cycles limite dont la période et l'amplitude sont peu différentes de celles qui sont observées.

Ce sont ces deux mémoires qui constituent les bases sur lesquelles s'est fondée ma théorie générale de la dynamique monétaire dans tous ses développements ultérieurs.

- De 1965 à 1987, dans différents mémoires, j'ai étendu les résultats précédents en spécifiant les fonctions de demande et d'offre de monnaie à partir d'une formulation entièrement nouvelle. Cette formulation est héréditaire en ce sens qu'elle détermine le présent comme une fonctionnelle du passé, et elle est relativiste en ce sens que cette dépendance est invariante lorsque l'on substitue à la considération du temps physique celle du temps psychologique. Elle considère qu'on oublie le passé comme on escompte l'avenir.

Il résulte de là qu'à un instant donné la valeur commune des taux d'oubli et d'intérêt est elle-même une fonctionnelle invariante des variations passées de la dépense globale.

- Actuellement, ma théorie de la dynamique monétaire repose sur quatre piliers : l'équation fondamentale de la dynamique monétaire, et les trois formulations héréditaires et relativistes de la demande de monnaie, de l'offre de monnaie, et des taux d'oubli et d'intérêt psychologique. 
Elle repose essentiellement sur des idées directrices originales et applicables dans de très nombreux domaines, en économie, en psychologie, en sociologie et en science politique :-l'analogie fondamentale entre l'oubli du passé et l'actualisation de l'avenir ; - le processus psychologique héréditaire de l'oubli ;- la considération du temps psychologique ; - le conditionnement héréditaire des hommes par les événements passés ; - la propagation héréditaire des phénomènes monétaires avec un affaiblissement progressif au cours du temps ; - la conception d'une régulation retardée impliquant l'existence de cycles limite.

Elle se fonde sur l'introduction de nouveaux concepts sans équivalent dans la littérature antérieure: - les concepts de taux d'intérêt psychologique, de taux d'oubli et de temps de réaction dont les valeurs varient avec la conjoncture ; - le concept de coefficient d' expansion psychologique qui traduitl'appréciation moyenne de la conjoncture par l'ensemble des agents économiques ; - le concept de temps psychologique, le référentiel de temps psychologique étant tel que les lois de la dynamique monétaire y soient invariantes.

- Dès lors que l'on dispose d'une mesure appropriée de la masse monétaire, les vérifications empiriques de la nouvelle théorie sont très remarquables. C'est je pense le seul cas dans toutel' histoire des recherches économétriques où un modèle ne faisant intervenir qu' une seule variable explicative et ne comportant que deux paramètres arbitraires, ou un seul suivant l'approche considérée, a pu donner, dans des cas aussi nombreux et aussi différents, de tels résultats, de très loin supérieurs à ceux de toutes les théories proposées antérieurement, ou depuis sa publication.

En mettant en évidence des effets invariants de type héréditaire et relativiste dans les phénomènes sociaux, la nouvelle approche ouvre de très larges perspectives, insoupçonnées jusqu'ici. Les résultats obtenus montrent que tout se passe comme si, indépendamment des cadres institutionnels différents, des situations historiques contingentes, et de leurs aspirations particulières, les hommes réagissaient de la même manière, et en quelque sorte mécaniquement, à des enchaînements complexes identiques. Ils montrent que nous sommes conditionnés par notre passé, et ils ouvrent de nouvelles perspectives dans le débat entre déterminisme et libre arbitre.

La théorie des probabilités, et l'analyse des séries temporelles et de leurs composantes exogènes

La réflexion sur la théorie des choix aléatoires, et la recherche des facteurs fondamentaux sous-jacents aux fluctuations des séries temporelles, et tout particulièrement à celles des résidus des modèles les mieux vérifiés, m'ont conduit à la fois à une analyse critique du concept de hasard et des théories des probabilités, à la démonstration d'un nouveau théorème, le Théorème $(T)$, et à la considération d'un nouveau concept, le $F$ acteur $X$, représentatif des influences physiques exogènes sur les séries temporelles. 
En fait les théories mathématiques que l'on désigne généralement par « théories mathématiques du hasard » ignorent le hasard, l'incertain, et la probabilité. Les modèles qu'elles considèrent sont des modèles purement déterministes, et, les quantités qu'elles étudient ne sont en dernière analyse que des fréquences de configurations particulières dans l'ensemble des configurations également possibles dont le calcul se fonde sur l'analyse combinatoire. En réalité aucune définition axiomatique du hasard n'est concevable.

Suivant l'hypothèse du «Facteur $X$ » les fluctuations des séries temporelles que nous observons dans les phénomènes qui relèvent des sciences de la nature, des sciences de la vie et des sciences de l'homme, résultent, pour une large part, de l'influence, par des effets de résonance, des innombrables vibrations qui sillonnent l'espace dans lequel nous vivons et dont l'existence est aujourd'hui une certitude. Ainsi peut s'expliquer, pour une large part, la structure des fluctuations, à première vue incompréhensible, que l'on constate dans un très grand nombre de séries temporelles comme par exemple celles des taches du soleil ou des cours de bourse. En fait ces fluctuations présentent toutes les apparences d'une structure presque périodique.

À une telle structure correspond une fonction presque périodique définie comme la somme de composantes sinusoïdales dont certaines périodes sont incommensurables. Il résulte du Théorème $(T)$ que sous des conditions très générales les valeurs successives d'une fonction presque périodique se distribuent suivant la loi normale. Il est ainsi établi que la structure déterministe vibratoire de l' univers peut entraîner des effets d'apparence aléatoire et que le déterminisme peut engendrer ce qu'il est communément convenu d'appeler le hasard.

\section{MA CONCEPTION DE LA SCIENCE ÉCONOMIQUE}

Telles sont les principales contributions, originales et novatrices, que je pense avoir apportées à la science économique fondamentale. Je voudrais maintenant résumer en quelques mots les principes que j'ai constamment suivis dans mes travaux depuis mon premier ouvrage de 1943.

\section{Le critère fondamental de l'expérience}

Tout d' abord, il n'y a de science que là où existent des régularités susceptibles d'être analysées et d'être prédites. Tel est par exemple le cas de la Mécanique céleste. Mais tel est également le cas d'une grande partie des phénomènes économiques. Leur analyse approfondie permet en effet de montrer l'existence de régularités tout aussi frappantes que celles que l'on constate dans les sciences physiques. C'est là la raison pour laquelle la discipline économique est une science, et pour laquelle cette science relève des mêmes principes et des mêmes méthodes que les sciences physiques.

Toute science repose sur des modèles, et tout modèle scientifique comporte trois stades bien distincts :- partir d'hypothèses bien explicitées ;- déduire de ces 
hypothèses toutes leurs conséquences et rien que leurs conséquences ;- confronter ces conséquences avec les données de l'observation. De ces trois phases, seules la première et la troisième, l'élaboration des hypothèses et la confrontation des résultats avec la réalité, présentent de l'intérêt pour l'économiste. La deuxième phase, purement logique et mathématique, c'est-à-dire tautologique, ne présente d'intérêt que mathématique.

Le modèle et la théorie qu'il représente doivent être acceptés, au moins provisoirement, ou rejetés, suivant qu'il y a accord ou désaccord entre les données de l'observation et les hypothèses et les implications du modèle. Une théorie dont ni les hypothèses ni les conséquences ne peuvent être confrontées avec le réel est dépourvue de tout intérêt scientifique. La seule déduction logique, fût-elle mathématique, si elle n'est pas étroitement rattachée à l'étude de la réalité, reste dépourvue de toute valeur quant à sa compréhension.

La soumission aux données de l'expérience est la règle d'or qui domine toute discipline scientifique. Toute théorie, quelle qu'elle soit, si elle n'est pas vérifiée par les données de l'expérience, n'a aucune valeur scientifique et elle doit être rejetée.

Tel est par exemple le cas des théories contemporaines de l'équilibre économique général qui se fondent sur l'hypothèse de convexité générale des champs de production, hypothèse contredite par toutes les données de l'expérience et qui entraîne des conséquences absurdes. Tel est encore le cas des théories néoBernoulliennes de l'utilité espérée qui reposent sur des postulats dont les conséquences sont incompatibles avec les données de l'observation.

Ma démarche s'est toujours fondée sur une double conviction :- la conviction que sans théorie la connaissance reste inéluctablement confuse, et qu'un amoncellement de faits ne constitue qu'un ensemble chaotique et inévitablement incompréhensibles ; - la conviction bien plus forte encore qu'une théorie qui ne peut être confrontée avec les faits, ou qui n'est pas vérifiée quantitativement par les données de l'observation, est en réalité dépourvue de toute valeur scientifique.

\section{L'illustration des théories par des modèles}

En second lieu, j'ai toujours illustré les théories générales que j'ai présentées par des modèles particuliers pour lesquels tous les calculs peuvent être explicités.

Ma conviction est en effet qu'aucune théorie générale ne peut être réellement comprise si elle n'est illustrée par la considération de modèles particuliers, judicieusement choisis de manière à pouvoir analyser toutes les circonstances intéressantes, et élaborés de manière à bien mettre en évidence les implications des hypothèses.

De même qu'il n'est pas de théorie scientifiquement valable qui ne soit générale et ne s'applique à tous les cas particuliers, de même il n'est pas de théorie générale qui puisse être pleinement comprise si elle n'est pas illustrée par son application à des cas particuliers. Plus une théorie est générale, plus son illustration par des modèles appropriés permet d'en comprendre pleinement la signification et la portée. 
La confrontation des théories avec les données empiriques et la recherche d' invariants

En troisième lieu, mes travaux ont été marqués par une préoccupation croissante des applications numériques à partir des données chiffrées fournies par l'observation.

- Les vérifications numériques auxquelles a donné lieu ma théorie héréditaire et relativiste de la dynamique monétaire sont tout à fait remarquables, à vrai dire les plus extraordinaires qui aient jamais été trouvées dans les sciences sociales, et cela dans un domaine essentiel pour la vie des sociétés. En fait la réalité observée est représentée d'une manière presque parfaite par la formulation à laquelle conduit cette théorie ; et cela qu'il s'agisse par exemple des États-Unis au cours de la Grande Dépression, de l'hyperinflation allemande de décembre 1919 à octobre 1923, au cours de laquelle l'indice des prix, sur la base 1913=100, a atteint la valeur de $10^{12}$, ou de l'hyperinflation soviétique de janvier 1922 à février 1924. Ces résultats démontrent l'existence dans les phénomènes économiques de régularités tout aussi frappantes que celles que l'on constate dans les sciences physiques.

- J'ai été peu à peu mené à une double conclusion : la psychologie des hommes reste fondamentalement la même en tout temps et en tout lieu; le présent est déterminé par le passé suivant des lois invariantes. Pour une très large part, comme dans les sciences physiques, l'élaboration des sciences sociales me paraît ainsi devoir reposer sur la recherche de relations et de quantités invariantes dans le temps et dans l'espace.

Ainsi, quelles que soient les économies considérées, qu'il s'agisse de celles des temps passés comme de celles d'aujourd'hui, toute l'activité économique des hommes se ramène à la recherche de surplus, à leur réalisation et à leur répartition suivant des processus fondamentalement invariants.

Ma théorie des processus intertemporels met en évidence une structure invariante quant à la relation existant entre la production à un moment donné et les facteurs de production fournis dans le passé qui peuvent être considérés comme à l'origine de cette production.

L'analyse des réponses au sondage que j'ai effectué en 1952 m'a amené à la constatation qu'il existe une utilité cardinale, et que pour tous les sujets cette utilité cardinale peut se représenter par une fonction invariante des variations relatives de leur capital.

La théorie de la dynamique monétaire que j'ai élaborée repose sur la considération d'une liaison héréditaire, invariante dans le temps et dans l' espace, entre le présent et l'évolution passée. Les résultats qu'elle a permis d'obtenir montrent que des sociétés humaines, situées dans des contextes très différents, qu'il s'agisse de situations courantes, inflationnistes ou déflationnistes, ou d'hyperinflations, de pays capitalistes ou communistes, d'aujourd'hui ou d'il y a cent ans, se comportent de manière semblable. Ainsi peut se fonder l'étude de notre conditionnement par 
le passé, et la formulation héréditaire et relativiste à laquelle je suis arrivé est susceptible de multiples applications dans tous les domaines des sciences humaines.

\section{L'utilisation des mathématiques}

En quatrième lieu, j'ai été constamment amené à utiliser les mathématiques dans tous les cas où la logique ordinaire ne pouvait manifestement suffire pour l'analyse des phénomènes économiques, par essence quantitatifs et souvent très complexes. Cette utilisation m'a permis de donner des solutions rigoureuses à des problèmes qui autrement auraient été insolubles en raison de leur complexité.

Cependant, les mathématiques ne sont et ne peuvent être qu' un outil, et tous mes travaux sont inspirés par cette conviction que dans leur utilisation les deux étapes vraiment fructueuses de la démarche scientifique sont, d'une part l'examen approfondi des hypothèses de départ, et d'autre part, la discussion de la signification des résultats obtenus au regard des données empiriques. Le reste n'est que calcul tautologique qui n'a d'intérêt que pour le mathématicien, et la rigueur mathématique des raisonnements ne saurait en aucun cas justifier une théorie fondée sur des postulats si ces postulats ne répondent pas à la nature réelle des phénomènes étudiés.

En aucun cas l'emploi des mathématiques les plus élevées ne doit être considéré comme une garantie de qualité. Les mathématiques ne sont et ne peuvent être qu'un moyen d'expression et de raisonnement. La substance même sur laquelle l'économiste travaille reste économique et sociale. En fait, il faut absolument éviter le développement d'un appareil mathématique complexe lorsqu'il n'est pas strictement indispensable. Le véritable progrès ne consiste jamais dans l'exposé purement formel, il consiste toujours dans la découverte des idées directrices qui sont à la base de toute démonstration. Ce sont ces idées de base qu'il convient d'expliciter et de discuter.

Les mathématiques ne sauraient constituer un but en soi ; elles ne sont et elles ne peuvent être qu'un moyen.

\section{Les idées nouvelles et la tyrannie des doctrines dominantes}

Enfin je n'ai jamais hésité à remettre en question les théories communément acceptées lorsqu'elles me sont apparues comme reposant sur des hypothèses impliquant des conséquences incompatibles avec les données de l'observation.

Ce n'est en fait que par la constante remise en cause des "vérités établies » et par la floraison d'idées nouvelles suggérées tant par l'expérience que par l'intuition créatrice que la science peut réellement progresser. Mais tout progrès scientifique réel se heurte à la tyrannie des idées dominantes et des « establishments » dont elles émanent. Plus les idées dominantes sont répandues, plus elles se trouvent en quelque sorte enracinées dans la psychologie des hommes, et plus il est difficile de 
faire admettre une conception nouvelle, si féconde qu'elle puisse se révéler ultérieurement.

Les idées dominantes, si erronées qu'elles puissent être, finissent par acquérir, par leur simple et incessante répétition, le caractère de "vérités établies » qu'on ne saurait mettre en doute sans s'opposer à l'ostracisme actif des " establishments ». L'exemple des Copernic, des Galilée, des Abel, des Galois, des Pasteur, des Arrhenius, des Wegener, et de bien d'autres encore, est là pour nous montrer à quels obstacles peuvent se heurter les découvreurs de génie.

C'est cette résistance aux idées nouvelles qui en économie explique qu'il ait fallu tant de temps pour que l'on découvre les apports majeurs de Dupuit, de Walras, d'Edgeworth, de Pareto, et de bien d'autres. Les Éléments d'Économie Pure de Walras n'ont été traduits en anglais que soixante-quinze ans après leur parution. Il n'a pas fallu moins de soixante ans pour que le Manuel d'Économie Politique de Pareto paraisse en langue anglaise.

Le savant à succès est toujours celui qui apporte quelque perfectionnement marginal aux théories dominantes auxquelles tout le monde est habitué. Si par contre une théorie est élaborée qui s'écarte des chemins battus, elle est assurée d'une opposition générale quelles que puissent être ses justifications.

Pour toutes ces raisons, il est essentiel de soumettre constamment les " vérités établies " à une analyse critique sans complaisance, en se rappelant sans cesse le jugement de Pareto : "L' histoire de la science se réduit à l' histoire des erreurs des hommes compétents ».

Quoi qu'il puisse lui en coûter, l'homme de science ne doit jamais se déterminer en fonction des modes du moment et de l'approbation, ou non, de ses contemporains. Sa seule préoccupation doit rester la recherche de la vérité. C'est là un principe dont je ne me suis jamais départi.

Je voudrais, pour terminer, vous exprimer une nouvelle fois ma reconnaissance pour la très haute distinction qui m'a été attribuée.

Comme l'a écrit autrefois Walras, il est bien certain que le vrai savant poursuit la vérité pour elle-même, mais il ne saurait être insensible à la reconnaissance de la valeur de son oeuvre. Quoi qu'ils aient pu en dire, les plus grands hommes de science ne sont jamais restés tout à fait indifférents à l'opinion des autres.

\section{BIBLIOGRAPHIE}

THÉORIE DE L'ÉVOLUTION ET DE L'ÉQUILIBRE ÉCONOMIQUE GÉNÉRAL, DE L'EFFICACITÉ MAXIMALE, ET DES FONDEMENTS DU CALCUL ÉCONOMIQUE

1943 À la recherche d' une Discipline Économique - Première Partie - L'Économie Pure 
Première édition, Ateliers Industria, 1943, 852 p. et Annexes, 68 p.

Deuxième édition, publiée avec le concours du Centre National de la Recherche Scientifique, sous le titre :Traité d'Économie Pure, Imprimerie Nationale, 5 vol., in $4^{\circ}, 984$ p. Cette seconde édition ne diffère de la première que par l'addition d'une Introduction à la deuxième édition (63 p.)

Troisième édition, Montchrestien, 1989.

1945 Économie Pure et Rendement Social

Paris, Sirey, 1945, 72 p.

1967 Les Conditions de l'Efficacité dans l'Économie

Rapport présenté au Quatrième Séminaire International organisé par le Centro Studi e Ricerche su Problemi Economico-Sociali de Milan, 12-14 Sept. 1967, 145 p.

École Nationale Supérieure des Mines de Paris, 1967.

1968 The Conditions of Efficiency in the Economy

Economia Internazionale, Vol. XXI, nº 3, Gênes, p. 3-24.

1971 Les Théories de l'Équilibre Économique Général et de l'Efficacité Maximale - Impasses Récentes et Nouvelles Perspectives

Revue d'Économie Politique, Mai-Juin 1971, n 3, p. 331-409.

1973 La Théorie Générale des Surplus et l'Apport Fondamental de Vilfredo Pareto

Revue d'Économie Politique, Nov.-Déc. 1973, nº 6, 83 année, p. $1044-$ 1097.

1973* The General Theory of Surplus and Pareto's Fundamental Contribution

Convegno Internationale Vilfredo Pareto (Roma, 25-27 Ottobre 1973), Roma, Accademia Nazionale dei Lincei, 1975, p. 109-163, (Traduction anglaise de Allais, 1973).

1974 Theories of General Economic Equilibrium and Maximum Efficiency

Dans l'ouvrage collectif : Equilibrium and Disequilibrium in Economic Theory, D. Reidel Publishing Company, Dordrecht, 1977, p. 129-201 (Traduction anglaise de Allais, 1971. La version anglaise ne diffère de la version française que par quelques additions marginales, notamment dans la note 18$)$.

1975 Les Implications de Rendements Croissants et Décroissants sur les Conditions de l'Équilibre Économique Général et d' une Efficacité Maximale

Contribution à l'ouvrage collectif : Hommage à François Perroux, Presses Universitaires de Grenoble, 1978, p. 605-674. 
1978 La Théorie Générale des Surplus

Économies et Sociétés, Paris, Janvier-Mai 1981, Institut de Sciences Mathématiques et Économiques, 2 vol., 718 p.

Seconde édition : Presses Universitaires de Grenoble, 1989.

1984 The Concepts of Surplus and Loss, and the Reformulation of the General Theory of Economic Equilibrium and Maximum Efficiency

Mémoire publié dans le volume Production and Exchange, The Foundations of Economic Knowledge, M. Baranzini et R. Scazzieri, éditeurs, Blackwell, Oxford, 1985, p. 135-174.

1987 The Equimarginal Principle. Meaning, Limits and Generalization.

Rivista Internazionale di Scienze Economiche E Commerciali, Vol. 34, 1987, N. 8, p. $689-750$

1987 Economic Surplus and the Equimarginal Principle

The New Palgrave. A Dictionary of Economics, Macmillan, Vol. 2, p. 62-69, 1987.

THÉORIE DES PROCESSUS INTERTEMPORELS ET D'UNE STRUCTURE CAPITALISTIQUE OPTIMALE

1947 Économie et Intérêt

Imprimerie Nationale et Librairie des Publications Officielles, Paris, 800 pages en deux volumes, 1947.

Deuxième édition, Montchrestien 1989.

1960 Influence du Coefficient Capitalistique sur le Revenu Réel par Tête

Mémoire présenté au Congrès de Tokyo (Juin 1960) de l'Institut International de Statistique, Document 61, 70 p., et Tome XXXVIII, 2, Tokyo, 1961, p. 3-27.

1961 The Influence of the Capital Output Ratio on Real National Income

Walras Bowley Lecture, American Meeting of the Econometric Society, New York, 28 Décembre 1961, Econometrica, Vol. 30, n 4, Octobre 1962.

Ce mémoire a été publié dans les Readings in Welfare Economics, American Economic Association, Vol. XII, 1969, p. 682-714, avec une "Additional Note », p. 711-714.

1963 Some Analytical and Practical Aspects of the Theory of Capital

Mémoire présenté au Congrès de Cambridge de l'Association Économique Internationale (Juil. 1963)

Publié dans l'ouvrage collectif : Activity Analysis in the Theory of Growth and Planning, édité par E. Malinvaud et M. Bacharach, MacMillan, Londres et St Martin's Press, New York, 1967, p. 64-107. 
1963 The Role of Capital in Economic Development

Mémoire présenté au cours de la Semaine d'Études sur le Rôle de l'Analyse Économétrique dans la Formulation de Plans de Développement, organisé par l'Académie Pontificale des Sciences (Rome, 7-13 Octobre 1963)

Publié dans le volume le Rôle de l'Analyse Économique dans la Formulation de Plans de Développement, Pontificiae Academiae Scientarium Scripta Varia, 28, Pontifica Academia Scientarium, 1965, Vol. II, p. $697-1002$.

Publié également dans The Econometric Approach to Development Planning, North Holland Publishing $\mathrm{C}^{\circ}$, Amsterdam et Rand Mac Nally, Chicago, 1965 , p. 697-1002.

1966 Produit National Brut Réel, Revenu National Réel et Fonction de CobbDouglas

Mémoire publié dans l'ouvrage collectif La Statistica Come Methodologia Delle Science Sociali, en l'honneur du Professeur Gini, Institut de Statistique, Rome 1966. Et aussi : Metron, Vol. XXV, n 1-4, 31 XII 1966, p. 310-355.

THÉORIE DES CHOIX ALÉATOIRES ET DES CRITÈRES À CONSIDÉRER POUR DES DÉCISIONS RATIONNELLES

1952 La Généralisation des Théories de l'Équilibre Économique Général et du Rendement Social au Cas du Risque

Mémoire présenté au Colloque International de Paris du Centre National de la Recherche Scientifique sur les «Fondements et Applications de la Théorie du Risque en Économétrie ", Paris, 12-17 Mai 1952.

Ce mémoire a été publié dans le volume collectif Économétrie, Collection des Colloques Internationaux du Centre National de la Recherche Scientifique, Vol. XL, Paris, 1953, p. 81-120.

1952 Fondements d'une Théorie Positive des Choix comportant un Risque et Critique des Postulats et Axiomes de l'École Américaine

Mémoire d'ensemble rédigé à la suite des débats du Colloque International de Paris.

Ce mémoire a été republié en 1955 avec quelques additions mineures dans un numéro spécial des Annales des Mines et sous la forme d'un volume séparé par l'Imprimerie Nationale ( $56 \mathrm{p}$. in $\left.4^{\circ}\right)$.

1952 La Psychologie de l'Homme Rationnel devant le Risque. La Théorie et l'Expérience

Journal de la Société de Statistique de Paris, Janvier- Mars 1953, p. 47-73. 
1952 L'Extension des Théories de l'Équilibre Économique Général et du Rendement Social au Cas du Risque

Econometrica, Vol. 21, n 2 , Avril 1953, p. 269-290.

1952 Le Comportement de l'Homme Rationnel devant le Risque. Critique des Postulats et Axiomes de l'École Américaine

Econometrica, Vol. 21, n 4, Octobre 1953, p. 503-546.

1979 Expected Utility Hypothesis and the Allais' Paradox; Contemporary Discussions of Decisions under Uncertainty with Allais' Rejoinder, Maurice Allais and Ole Hagen editors, Reidel Publishing Company, Dordrecht, $1979,714 \mathrm{p}$.

Contributions personnelles :

1. «Foreword» (p. 3-11)

2. "The Foundations of a Positive Theory of Choice Involving Risk and a Criticism of the Postulates and Axioms of the American School » (p. 25145).

Traduction anglaise du mémoire « Fondements d'une théorie positive des choix comportant un risque et critique des postulats et axiomes de l'école américaine », Vol. XL, Économétrie, Colloques Internationaux du Centre National de la Recherche Scientifique, Paris, 1953.

3. "The So-called Allais' Paradox and Rational Decisions under Uncertainty » (p. 434-698).

1984 The Foundations of the Theory of Utility and Risk

Dans l'ouvrage "Progress in Decision Theory », édité par O. Hagen et F. Wenstop, D. Reidel Publishing Company, Dordrecht, 1984, p. 3-131.

1984 Determination of Cardinal Utility according to an Intrinsic Invariant Model

Dans l'ouvrage collectif "Recent Development in the Foundations of Utility and Risk Theory », p. 83-120, L. Daboni et al édit., (Proceedings of the 1984 Venice Conference), D. Reidel Publishing Compagny, 1986.

1985 Three Theorems on the Theory of Cardinal Utility and Random Choice

In Essays in honour of Werner Leinfellner, Theory and Decision, Gerald Heberlein and Hal Berghel, édit., p. 205-221, D. Reidel Publishing Compagny, Dordrecht, 1987.

1985 The Allais Paradox

The New Palgrave. A Dictionary of Economics, Vol. 1, p. 78-80, MacMillan, 1987.

1986 The General Theory of Random Choices in Relation to the Invariant 
Cardinal Utility Function and the Specific Probability Function. The $(U, \theta)$ Model. A General Overview

Dans l'ouvrage collectif «Risk, Decision and Rationality », Bertrand Munier, édit., D. Reidel Publishing Company, Dordrecht, 1987,p. 231-289.

1986 A New Neo-Bernoullian Theory : The Machina Theory-A Critical Analysis

Dans l'ouvrage collectif "Risk, Decision and Rationality », Bertrand Munier, édit., D. Reidel Publishing Company, Dordrecht, 1987,p. 347-403.

1988 Cardinal Utility. History, Empirical Findings and Applications

Fourth International Conference on the Foundations and Applications of Utility, Risk, and Decision Theory, Budapest, Juin 1988, Kluwer Publishing Company, 1989.

1989 Scientific Papers on Risk and Utility Theory. Theory, Experience, and Applications

Kluwer Publishing Company, 1.000 p.

THÉORIE DE LA MONNAIE, DU CRÉDIT, ET DE LA DYNAMIQUE MONÉTAIRE

1954 Les Fondements Comptables de la Macroéconomique - Les Équations Comptables entre Quantités Globales et leurs Applications

Presses Universitaires de France, Paris, 1954.

Deuxième édition, 1989.

1954 Explication des Cycles Économiques par un Modèle non Linéaire à Régulation Retardée

Metroeconomica, Vol. VIII, Avril 1956, fascicule 1, p. 4-83.

1955 Explication des Cycles Économiques par un Modèle non Linéaire à Régulation Retardée, Mémoire complémentaire

Mémoire publié dans le volume collectif : Les Modèles Dynamiques en Économétrie, Collection des Colloques Internationaux, Centre National de la Recherche Scientifique, Vol. LXII, Paris, 1956, p. 259-308.

1965 Reformulation de la Théorie Quantitative de la Monnaie

Éditions SEDEIS (205 Bld Saint-Germain, Paris, $7^{\mathrm{e}}$ ), in- $4^{\mathrm{o}}$, Septembre $1965,186 \mathrm{p}$.

1965 Reformulation de la Théorie Quantitative de la Monnaie

Bulletin de l'Institut International de Statistique, Actes de la $35^{\mathrm{e}}$ Session, Tome XLI, $2^{\mathrm{e}}$ livraison, 1966, p. 905-947. 
1966 A Restatement of the Quantity Theory of Money

American Economic Review, Vol. LVI, no 5, Décembre 1966, p. 1123 1156.

1968 Growth and Inflation

American Bankers Association, Conference of University Monetary Economists, Ditchley Park, 10-13 Septembre 1968.

Journal of Money, Credit and Banking, Août 1969, p. 355-426 et 427 462.

1972 Forgetfulness and Interest

Journal of Money, Credit and Banking, Février 1972, p. 40-71.

1973 Le Concept de Monnaie, la Création de Monnaie et de Pouvoir d' Achat par le Mécanisme du Crédit et ses Implications dans « Monnaie et Développement », Vol.4,p. 5-104, École Nationale Supérieure des Mines (E.N.S.M.P.), Paris, 1975

Des extraits ont été publiés dans : Essais en l' honneur de Jean Marchal, Paris, Cujas, 1975, Vol. 2, La Monnaie, p. 106-145.

1974 The Psychological Rate of Interest

Journal of Money, Credit and Banking, Août 1974, p. 285-331.

1980 La Formulation Héréditaire et Relativiste de la Demande de Monnaie et du Taux d'Intérêt

Conférence et Séminaire des 5 et 6 Mai 1980, Département d’Économétrie de l'Université de Genève. Texte reproduit dans Allais, Fondements de l'Analyse Monétaire et Conjoncturelle ; V, p. 105-177, E.N.S.M.P., 1980.

1984 The Credit Mechanism and its Implications

Contribution à l'ouvrage collectif : Arrow and the Foundations of the Theory of Economic Policy, edited by George R. Feiwell, p. 491-561, MacMillan Press, 1987, 758 p.

1984 ANew Empirical Approach of the Hereditary and Relativistic Theory of the Demand for Money

Dans « Articole in Memoria di Tullio Bagiotti » A. Agnati, D. Cantarelli, et A. Montesano, édit., Rivista Internazionale di Scienze Economiche e Commerciali, Oct..-Nov. 1985, $\mathrm{n}^{\text {os }}$ 10-11, p. 905-948.

1985 The Empirical Approaches of the Hereditary and Relativistic Theory of the Demand for Money

Economia della Scelte Pubbliche, Journal of Public Finance and Public Choice (Fondazione Luigi Einaudi), 1986, p. 3-83. 
LA THÉORIE DES PROBABILITÉS, ET L'ANALYSE DES SÉRIES TEMPORELLES ET DE LEURS COMPOSANTES EXOGÈNES

1957 Test de Périodicité - Généralisation du Test de Schuster au cas de Séries Temporelles Autocorrélées

Comptes-Rendus de l'Académie des Sciences, Tome 244, 13 Mai 1957, no 20 , p. $2469-2471$.

1959 Test de Périodicité - Généralisation du Test de Schuster au cas de Séries Temporelles Autocorrélées

Vol. 1 des Studi in Onore di Corrado Gini, Istituto di Statistica della Facoltà di Scienze Statistische Demografiche ed Attuariali, p. 1-14.

1961 Généralisation du Test de Schuster au cas de Séries Temporelles Autocorrélées dans l' Hypothèse d' un Processus de Perturbations Aléatoires d' un Système Stable

Communication au $33^{\mathrm{e}}$ Congrès de l'Institut International de Statistique, Paris, 1961, Bulletin de l'Institut International de Statistique, 1962, Tome 39, $2^{\mathrm{e}}$ livraison, p. 143-194.

1977 On the Concept of Probability

Rivista Internazionale di Scienze Economiche e Commerciali, Novembre $1978, \mathrm{n}^{\circ} 11$, p. $937-956$.

1982 Frequency, Probability and Chance

Dans l'ouvrage Foundations of Utility and Risk Theory with Applications édité par Bernt F. Stigum et Fred Wenstop, D. Reidel Publishing Company, Dordrecht, 1983 , p. 35-84.

1983 Sur la Distribution Normale des Valeurs à des Instants régulièrement Espacés d' une Somme de Sinusoïdes

Communication à l'Académie des Sciences, 30 Mai 1983, ComptesRendus de l'Académie des Sciences, Tome 296, Série 1, p. 829-832.

1983 Fréquence, Probabilité et Hasard

avec deux Appendices :

1. Fréquences empiriques et fréquences mathématiques - Illustration

2. Le théorème $(T)$-La simulation du hasard par des fonctions presque périodiques

Journal de la Société de Statistique de Paris, 2e et 3e trimestre 1983, p. 70-102 et 144-221.

1987 Some Remarkable Properties of the Determination of a Continuous Distribution by its Moments

Dans l'ouvrage collectif «Risk, Decision and Rationality », Bertrand Munier, édit., D. Reidel Publishing Company, 1987, p. 557-561. 
1988 Phénomènes Aléatoires et Modèles Fréquentiels. Réalité et Théorie. Prolégomènes pour une Révision des Théories Admises.

Conférence du 5 octobre 1988, Rheinisch Westfähsche Akademic des Wissenchaften.

VUES D'ENSEMBLE SUR MES CONTRIBUTIONS SCIENTIFIQUES

1978 Contributions à la Science Économique - Vue d'ensemble 1943-1978

Paris, Centre d'Analyse Économique, 176 p.

1979 Sur mes Travaux 1943-1979

Paris, Centre d'Analyse Économique, 19 p.

1979 Discours prononcé le 13 Mars 1979 à l'occasion de la remise de la Médaille d'Or du Centre National de la Recherche Scientifique par Madame Alice Saunier-Seité, Ministre des Universités

Revue d'Économie Politique, Juillet-Août 1979, p. 553-563.

1986 Principaux Ouvrages et Mémoires, 1943-1985

Centre d'Analyse Économique, 42 p.

1986 Essais en l' honneur de Maurice Allais - Marchés, Capital et Incertitude

Economica, Paris, 1986, 265 p., Marcel Boiteux, Thierry de Montbrial, Bertrand Munier édit.

- Présentation par Marcel Boiteux, Thierry de Montbrial et Bertrand Munier, p. 5-9 ;-L'œuvre d'économiste de Maurice Allais, Achèvements théoriques et germes du renouveau, Bertrand Munier, p. 13-37 ;-Maurice Allais, savant méconnu, Thierry de Montbrial, p. 39-44.

Une traduction anglaise de cet ouvrage sera publiée par les Éditions Kluwer Academic Publishers, Dordrecht, en 1989, sous le titre, "Markets, Capital, and Uncertainty. Essays in Honour of Maurice Allais ». 